\title{
Isolamento de Cryptococcus neoformans, C. gattii e C. Iaurentii de sistema nervoso central de cães na cidade do Rio de Janeiro, RJ, Brasil*
}

\author{
Isolation of Cryptococcus neoformans, C. gattii and C. laurentii \\ from central nervous system of dogs in Rio de Janeiro City, RJ, Brazil \\ Felipe Lopes Campos', Juan Rojas Pereira², Marlon Vicente da Silva ${ }^{3}$, \\ Beatriz Cristina Pereira dos Santos ${ }^{3} \&$ Francisco de Assis Baroni ${ }^{4}$
}

\begin{abstract}
RESUMO
A criptococose é uma micose sistêmica com caráter oportunista, que pode acometer homens e diferentes espécies animais, principalmente em casos de imunodepressão. A infecção pode ser adquirida através da inalação, e por via hematógena, tem a capacidade de atingir e colonizar o sistema nervoso central. Objetivando o isolamento da levedura Cryptococcus neoformans a partir de sistema nervoso central de cães e gatos, no Município do Rio de Janeiro, procederam-se coletas no Laboratório de Diagnóstico de Raiva do Instituto Municipal de Medicina Veterinária Jorge Vaitsman, Rio de Janeiro. A etapa de isolamento e identificação se deu no Laboratório de Leveduras Patogênicas e Ambientais da Universidade Federal Rural do Rio de Janeiro (LLPA-UFRRJ). Obteve-se um total de duzentas e uma amostras, sendo 166 de cães e 35 de gatos, resultando em quatro isolamentos positivos para o gênero nas amostras provenientes de cães; sendo um de Cryptococcus laurentii, dois de Cryptococcus neoformans e um de Cryptococcus gattii. Com este trabalho, obteve-se o primeiro isolamento descrito de Cryptococcus laurentii de sistema nervoso central de cão.
\end{abstract}

Descritores: Cryptococcus neoformans, Cryptococcus laurentii, Cryptococcus gattii, sistema nervoso central.

\section{ABSTRACT}

The cryptococcosis is an opportunistic systemic mycosis, which can affect humans and various animal species, especially in cases of immunosuppression. The infection can be acquired through inhalation and by hematogenous route has the ability to reach and colonize the central nervous system. The aim of this survey was the isolation of the Cryptococcus neoformans yeast from central nervous system of dogs and cats in Rio de Janeiro City. Periodic collections were made from brain samples at the rabies diagnosis laboratory in Instituto Municipal de Medicina Veterinária Jorge Vaitsman (IJV), Rio de Janeiro, Brazil. The isolation and the identification of this yeast were performed at the Laboratory of environmental and pathogenic yeasts of the Universidade Federal Rural do Rio de Janeiro (UFRRJ). According to the results, of the 201 clinical samples (166 samples from dogs and 35 from cats), we found four positives samples; one of these was positive for C. laurentii, two were positive for Cryptococcus neoformans; and one Cryptococcus gattii. In this paper we reported the first isolation of Cryptococcus laurentii from the central nervous system of dogs.

Keywords: Cryptococcus neoformans, Cryptococcus laurentii, Cryptococcus gattii, central nervous system.

*Artigo originado de Dissertação de Mestrado do primeiro autor. Pesquisa financiada pela Coordenação de Aperfeiçoamento de Pessoal de Nível Superior (CAPES). 'Universidade Estadual do Centro-Oeste (UNICENTRO), Rua Salvatore Renna-Padre Salvador n.875, Santa Cruz, CEP 85015-430 Guarapuava, PR, Brasil. ${ }^{2}$ Médico Veterinário Autônomo. ${ }^{3}$ Instituto Municipal de Medicina Veterinária Jorge Vaitsman (IJV), Rio de Janeiro, RJ, Brasil ${ }^{4}$ Departamento de Microbiologia e Imunologia Veterinária, Universidade Federal Rural do Rio de Janeiro (UFRRJ), Seropédica, RJ, Brasil. CORRESPONDÊNCIA: F.L. Campos [campos.79@gmail.com - Fax: + (55) 423621 1034]. 


\section{INTRODUÇÃO}

A criptococose é uma micose sistêmica e oportunista que pode acometer homens e diferentes espécies animais, principalmente em casos de imunodepressão. A levedura Cryptococcus neoformans é encontrada em todo o mundo, em diversos tipos de solos e em tecidos, secreções e excreções de animais e no próprio homem $[3,5,10,11,13,29]$.

A infecção é adquirida através da inalação de esporos e leveduras, e por via hematógena $[18,19,28$, 29], podendo atingir o sistema nervoso central (SNC), $[5,19,20,31,32]$. Os sinais neurológicos estarão presentes de acordo com o envolvimento das meninges ou com aumento da pressão intracraniana [30].

Em cães, o sistema nervoso pode ser afetado [17,20,22,24,29,35], promovendo sinais variáveis de acordo com a localização das lesões, que podem ocorrer nas meninges, no encéfalo, na medula, nos nervos periféricos [17,29]. Os sinais clínicos mais comuns são ataxia, depressão, paresia, convulsão, andar em círculo, perda de olfato e cegueira.

Em gatos, a criptococse foi descrita pela primeira vez em 1971 [6]. O Cryptococcus neoformans pode afetar os diferentes órgãos e tecidos, como o trato respiratório, a pele, o SNC e os olhos $[5,15,24,29$, 32]. A cavidade nasal e os seios nasais são os mais afetados [14], com a formação de pólipos, dando o aspecto de "nariz de palhaço" [13,32].

Com este trabalho, objetiva-se isolar Cryptococcus neoformans de SNC de cães e gatos, com sinais neurológicos e encaminhados ao Laboratório de Diagnóstico de Raiva do Instituto Municipal de Medicina Veterinária Jorge Vaitsman, Rio de Janeiro.

\section{MATERIAIS E MÉTODOS}

A primeira parte do projeto foi desenvolvida junto ao Laboratório de Diagnóstico de Raiva do Instituto Municipal de Medicina Veterinária Jorge Vaitsman (IMJV), que recebe periodicamente materiais oriundos do sistema nervoso central de animais domésticos e silvestres. As amostras coletadas para o desenvolvimento desta pesquisa eram provenientes de sistema nervoso central de cães e gatos, englobando cérebro, cerebelo e bulbo. As etapas de isolamento e identificação do Cryptococcus spp. foram realizadas no Laboratório de Leveduras Patogênicas e Ambientais do Departamento de Microbiologia e Imunologia Veterinária da UFRRJ (LLPA-UFRRJ).
Obteve-se do IMJV, no período de janeiro de 2004 a julho de 2005, duzentas e uma amostras de SNC, das quais 166 eram provenientes de cães (76 machos e 90 fêmeas) e 35 de gatos (19 machos e 16 fềmeas), de ambos os sexos, com idades entre dois meses e doze anos. Previamente ao isolamento, o material triturado foi assepticamente colocado no interior de frascos de Erlenmeyer, com salina estéril $(200 \mathrm{~mL} /$ frasco $)$, acrescida de cloranfenicol $(100 \mathrm{mg} / \mathrm{L})$, passando por agitação magnética por dez minutos e sedimentação por uma hora [2,3]. O sobrenadante foi aspirado e $0,1 \mathrm{~mL}$ foi inoculado em triplicata, utilizando-se placa de Petri com meio Sabouraud dextrose $4 \%^{1}$ e duas placas de Petri contendo meio com dopamina [8,26,34], ambos os meios de isolamento acrescidos de cloranfenicol.

As colônias suspeitas de Cryptococcus (lisas, úmidas, brilhantes e de coloração marrom-escura) foram novamente submetidas à prova de produção de fenoloxidase em meio com dopamina. Em sequência, foi realizado o teste de produção de urease em meio Christensen ${ }^{2}$, além de assimilação de açúcares (fontes carbonadas) e fontes nitrogenadas (auxanograma) e teste de fermentação negativa de açúcares (zimograma). O controle dos inoculados foi obtido com cepas de Cryptococcus neoformans (ICB 163) e Cryptococcus gattii (ICB 162) pertencentes ao acervo micológico do LLPA-UFRRJ.

Antes de serem submetidas aos testes, todas as cepas foram repicadas em meio Sabouraud dextrose a $4 \%$ e mantidas por 48 horas, para a obtenção de cultura jovem.

\section{RESULTADOS}

Das duzentas e uma amostras coletadas, obteve-se quatro isolamentos pertencentes ao gênero, todos na espécie canina (Tabela 1), sendo um isolado de Cryptococcus laurentii, dois de Cryptococcus neoformans e um de Cryptococcus gattii. Todos os isolados apresentaram reação positiva na prova de produção de urease.

\section{DISCUSSÃO}

A utilização de amostras de SNC de cães e gatos compatibiliza-se com o interesse de fazer um diagnóstico diferencial para síndromes neurológicas [29,35]. Pesquisadores têm descrito a ocorrência de Cryptococcus neoformans a partir de SNC em cães e gatos que apresentavam sintomatologia nervosa [20]. Neste trabalho, o mesmo agente foi encontrado apenas em SNC de cães. A síndrome neurológica dependerá 
Tabela 1. Isolados de Cryptococcus no Laboratório de leveduras patogênicas e ambientais da UFRRJ, a partir de amostras de sistema nervoso central*, relacionando espécie animal acometida, raça e sexo.

\begin{tabular}{cccc}
\hline Isolado & Espécie & Raça & Sexo \\
\hline Cryptococcus neoformans & Cão & Cocker Spaniel & Macho \\
Cryptococcus neoformans & Cão & SRD $^{1}$ & Macho \\
Cryptococcus gattii & Cão & SRD $^{1}$ & Macho \\
Cryptococcus laurentii & Cão & Dog alemão & Macho \\
\hline
\end{tabular}

*Amostras coletadas no Instituto Municipal de Medicina Veterinária Jorge Vaitsman na cidade do Rio de Janeiro, no período de 2004/2005. 'Sem raça definida.

da localização da lesão e observa-se principalmente, depressão, desorientação, vocalização, ataxia, espasticidade, cegueira, paresia, paraplegia, convulsão, anisocoria $[22,24,29]$.

Num estudo de 112 cães que apresentavam sinais neurológicos, respiratórios ou ambos, no Estado do Rio Grande do Sul, não foi obtido o isolamento da levedura [27], o que demonstra que outras causas podem originar sinais compatíveis com criptococose. Outros autores, ao trabalharem com um cão da raça labrador, diagnosticaram criptococose em caráter sistêmico e neurológico por citologia aspirativa, não preconizando o isolamento [23]. A similaridade entre os sinais neurológicos apresentados na criptococose com outros agentes infecciosos pode gerar um subdiagnóstico na rotina clínica. $\mathrm{O}$ diagnóstico precoce, baseado principalmente no isolamento, favorece a instituição da terapia e dos resultados preconizados [22].

Muitos relatos apresentam Cryptococcus neoformans como principal agente da criptococose humana e animal, com envolvimento respiratório ou nervoso [7,14]. Neste trabalho, obteve-se um isolado de Cryptococcus gattii, que apesar de já identificado em amostras clínicas como agente etiológico em humanos, animais silvestres e domésticos $[11,18]$, tem sido relatado com frequência em fontes ambientais $[1,19]$. Dessa forma, o contato com locais comumente implicados como reservatórios da levedura, como excretas de pombos $[2,3,10,28]$, pode ser um potencial fator de risco, principalmente para animais de companhia com condição corpórea insatisfatória [22].

O isolamento de Cryptococcus laurentii como agente causador de criptococose é raro. Em Lisboa, ao estudar 46 cães com quadro de otite por levedura, constatou-se que $13,1 \%$ apresentaram $C$. laurentii como agente etiológico [4]. Em aves da espécie Lamprotornis chalybaeus, essa levedura foi associada à perda de plumagem [9] e, em humanos, foi demonstrado um caso de meningoencefalite por $C$. laurentii em paciente com HIV [21]. Isolados a partir de amostras clínicas são pouco comuns e o isolamento a partir de sistema nervoso de cão ocorrido neste trabalho vem reforçar essa possibilidade etiológica para a criptococose no sistema nervoso, ressaltando a importância da identificação da levedura.

\section{CONCLUSÕES}

Sintomas neurológicos podem ser associados, além de outros fatores, à presença de Cryptococcus neoformans, Cryptococcus gattii e Cryptococcus laurentii.

Este trabalho pode ser considerado o primeiro a relatar o isolamento de Cryptococcus laurentii a partir de SNC de cão.

NOTAS INFORMATIVAS

${ }^{1}$ DIFCO Labratories ${ }^{\circledR}$, Detroit, EUA

${ }^{2}$ HIMEDIA Laboratories ${ }^{\circledR}$ Pvt. Ltda, Mumbai, Índia

\section{REFERÊNCIAS}

1 Baltazar L.M. \& Ribeiro M.A. 2008. Primeiro isolamento ambiental de Cryptococcus gattii no Estado do Espírito Santo. Revista Brasileira de Medicina Tropical. 41(5): 449-453.

2 Baroni F.A. 2001. Ocorrência de Cryptococcus neoformans em excretas de pombos localizados em torres de igrejas na cidade do Rio de Janeiro: fatores de virulência e sensibilidade aos antifúngicos. 150f. São Paulo, SP. Tese. (Doutorado em Microbiologia) - Curso de Pós-graduação em Microbiologia Universidade de São Paulo. 
3 Baroni F.A., Paula C.R., Silva E.G., Viani F.C., Rivera I.N.G., Oliveira M.T.B. \& Gambale W. 2006. Cryptococcus neoformans isolated from church towers in Rio de Janeiro city, RJ, Brazil. Revista do Instituto de Medicina Tropical de São Paulo. 78(2): 71-75.

4 Bernardo F.M., Martins H.M. \& Martins M.L. 1998. A survey of mycotic otitis externa of dos in Lisbon. Revista Iberoamericana de Micología. 15(3): 163-165.

5 Bichard S.J. \& Scerding R.G. 2008. Manual saunders de clínica de pequenos animais. 3.ed. São Paulo: Roca, 2048p.

6 Chagas W.A., Figueiredo J.B., Cruz L.C.H. \& Neves J.G. 1971. Criptococose cutânea em gato doméstico. Descrição de um caso. Revista Brasileira de Medicina Veterinária. 1: 5-12.

7 Chakrabarti A., Sharma A., Sood A., Grover R. \& Sakhuja V. 2000. Changing scenario of cryptococcosis in a tertiary care hospital in north India. Indian Journal of Medical Research. 112: 56-60.

8 Chaskes S. \& Tyndaall. 1975. Pigment production by Cryptococcus neoformans from para and ortho-diphenols effect of the nitrogen source. Journal of Clinical Microbiology. 1(6): 509-514.

9 Decostere A., Hermans K., De Baer T., Pasmans F. \& Haesebrouck F. 2003. First report on Cryptococcus laurentii associated with feather loss in a glossy starling (Lamprotornis chalybaeus). Avian Pathology. 32(3): 309-311.

10 Farias Filho L.P., Neves M.A., Souza-Castro G.B. \& Fortes S.T. 2004. Cryptococcus neoformans em excretas de pombos em Boa Vista/RR. In: Anais do IV Congresso Brasileiro de Micologia. (Ouro Preto, Brasil). p.45.

11 Fernandes O.F.L., Silva M.R.B., Ribeiro E.L., Costa M.R., Costa T.R., Silva M.V., Rodrigues A.B. \& Quirino H.M. 1999. Cryptococcus neoformans var. gattii isolamentos em pacientes com aids no estado de Goiás. Revista de Patologia Tropical. 28(1): 49-55.

12 Fernandes O.F.L., Costa T.R., Costa M.R., Soares A.J., Pereira A.J.S.C. \& Silva M.R.R. 2000. Cryptococcus neoformans isolados de pacientes com AIDS. Revista da Sociedade Brasileira de Medicina Tropical. 33(1): 75-78.

13 Grecco F.B. \& Moreno G. 2003. Cryptococose felina - relato de um caso em Cuiabá - Mato Grosso. In: Resumo do XI Encontro Nacional de Patologia Veterinária - ENAPAVE. (Botucatu, Brasil). p.124.

14 Hamilton A. 2002. Cryptococcus neoformans - the encapsulated menace. Mycologist. 16(3): 125-26.

15 Juliano R.S., Souza A.I. \& Scheid R. 2006. Criptococose felina - relato de caso. Revista de Patologia Tropical. 35(1): 65-70.

16 Kerl M.E. 2003. Update on canine and feline fungal disease. Veterinary Clinics: Small Animal Practice. $33(4)$ : $721-747$.

17 Kerwin S.C., McCarthy R.J., VanSteenhouse J.L., Partington B.P. \& Taboada J. 1998. Cervical spinal cord compression caused by cryptococcosis in a dog: succesful treatment with surgery and fluconazole. Journal of the American Animal Hospital Association. 34(6): 523-526.

18 Kidd S.E., Chow Y., Mak S., Bach P.J., Chen H., Hingston A.O., Kronstad J.A. \& Bartlentti K.H. 2007. Characterization of Environmental Sources of the Human and Animal Pathogen Cryptococcus gattii in British Columbia, Canada, and the Pacific Northwest of the United States. Applied and environmental microbiology. 73(5): 1433-1443.

19 Lazera M.S., Igreja R.P. \& Wanke B. 2004. Criptococose. In: Sidrim J.J.C. \& Rocha M.F.G. (Eds). Micologia médica à luz de autores contemporâneos. 2.ed. Rio de Janeiro: Editora Guanabara Koogan S.A., pp. 252-264.

20 Lavely J. \& Lipsitz D. 2005. Fungal infections of the central nervous system in the dog and the cat. Clinical Techniques in Small Animal Practice. 20(4): 212-219.

21 Manfredi R., Fulgaro C., Sabbatani S., Legnani G. \& Fasulo G. 2006. Emergence of amphotericin B-resistant Cryptococcus laurentii meningoencephalitis shortly after treatment for Cryptococcus neoformans meningitis in a patient with AIDS. AIDS Patient Care and STDs. 20(4): 227-232.

22 Marcasso R.A., Sierra S., Arias B.M.V., Bracarense A.P.R.F.L., Vamamura A.A.M., Biasi F., Lopes B.A., Amude A.M. \& Cortêz D.E.A. 2005. Criptococose no sistema nervoso de cães - relato de três casos. Semina: Ciências Agrárias. 26(2): 229-238.

23 Martins D.B., Barbosa A.L.T., Cavalheiro A., Lopes S.T.A., Santurio J.M., Schossler J.E. \& Mazzan A. 2008. Diagnóstico de criptococose canina pela citologia aspirativa por agulha fina. Ciência Rural. 38(3): 826-829.

24 Nelson R.W. \& Couto C.G. 2006. Medicina interna de pequenos animais. 3.ed. Rio de Janeiro: Elsevier, 1324p.

25 Norsworthy G.D., Crystal M.A., Grace S.F. \& Tilley L.P. 2004. O paciente felino. 2.ed. São Paulo: Manole, 815p.

26 Nuruden T.A. \& Ahearn D.G. 1979. Regulation of melanin production by Cryptococcus neoformans. Journal of Clinical Microbiology. 10(5): 724-729.

27 Oliveira I.A. \& Ferreiro L. 2005. Pesquisa de criptococose em cães atendidos no Hospital de Clínicas Veterinárias da UFRGS, Porto Alegre, Brasil. Acta Scientiae Veterinarie. 33(3): 253-258. 
28 Pappalardo M.C.S.M. \& Melhem M.S.C. 2003. Cryptococcosis: a review of the brazilian experience for the disease. Revista do Instituto de Medicina Tropical de São Paulo. 45(6): 299-305.

29 Pereira A.C.C. \& Coutinho S.D.A. 2003. Criptococose em cães e gatos - uma revisão. Clínica Veterinária. (45): $24-32$.

30 Queiroz J.P.A.F., Souza D.N., Lage R.A. \& Santos A.G. 2008. Criptococose - uma revisão. Acta Veterinária Brasílica. 2(2): 32-38.

31 Sá A.C.M., Rozemfeld A.C., Moura-Neto V., Souza W. \& Rozental S. 2004. Estudo da interação do fungo Cryptococcus neoformans com células microgliais. In: Anais do IV Congresso Brasileiro de Micologia. (Ouro Preto, Brasil). p.56.

32 Scott D.W., Kirk R.W. \& Muller G.H. 1995. Dermatologia de Pequenos Animais. 5.ed. Rio de Janeiro:Interlivros, 341p. 33 Silva E.G., Baroni F.A., Viani F.C., Ruiz L.S., Gandra R.F., Auler M.E., Dias A.L.T., Gambale W. \& Paula C.R. 2006. Virulence profile of Cryptococcus neoformans var. grubii evaluated by experimental infection in Balb/c mic and correlation with exoenzime activit. Journal of Medical Microbiology. 55: 139-142.

34 Staib F. 1962. Cryptococcus neoformans und Guizotia abyssinica (syn. G. oleifera D.C.) (Farbereaktion fur C. neofornans). Zeitschrift fur Hygiene. 148(5): 466-475.

35 Tiches D., Vite C.H., Dayrell-Hart B., Steinberg S.A., Gross S. \& Lexa F. 1998. A case of canine central nervous system cryptococcosis: management with fluconazole. Journal of the American Animal Hospital Association. 34(2): 145-151. 